American Journal of Applied Sciences 9 (9): 1378-1384, 2012

ISSN 1546-9239

(C) 2012 Science Publication

\title{
Discovery of an Equilibrium Circle in the Circular Restricted Three Body Problem
}

\author{
${ }^{1,2}$ Fawzy A. Abd El-Salam \\ ${ }^{1}$ Department of Math, Faculty of Science, Taibah University, Al-Madeenah, Saudi Arabia \\ ${ }^{2}$ Department of Astronomy, Faculty of Science, Cairo University, Cairo, 12613, Egypt
}

\begin{abstract}
Problem statement: A traial to find equilibrium points in out of plane of the restricted three body problem. Approach: Solution of the equations of motion at equilibrium points. Linearizing the equations of motion and computing the eigen values to investigate the stability. Results: New triangular equilibrium points in plane perpendicular to the plane of motion and passing through Lagrange triangular equilibrium points are obtained. A circle of equilibrium points, namely Fawzy equilibrium circle is discovered. Infinite number of equilibrium points located on the circumference of this circle are computed. The obtained solutions are checked via obtaining some special cases. The stability of Fawzy equilibrium circle is studied. The oscillatory stable solutions as $\eta$-dependent and $\xi$ dependent stabilities are derived. Conclusion: The equilibrium points in out of plane of motion of the restricted three body problem is investigated. We obtained the following very new results:- First we obtained Fawzy $\xi \xi$-triangular equilibrium points in the plane $\eta=0$. Second we obtained the so called Fawzy equilibrium circle. We checked our solutions via obtaining some special cases. We studied the stability of Fawzy equilibrium circle. We derived the oscillatory stable solutions as $\eta$-dependent and $\xi$ dependent stabilities. The stability of Lagrange as well as Fawzy triangular equilibrium points followed directly because they are subsets of the Fawzy equilibrium circle.
\end{abstract}

Key words: Fawzy equilibrium circle, stability, the three body problem

\section{INTRODUCTION}

In any assumed isolated two-body massive orbiting system (such as the Sun and the Earth) there are five equilibrium points, $\mathrm{L}_{\mathrm{i}}, \mathrm{i}=1,2,3,4,5$ these points usually called Lagrangian or libration points. At these points the gravitational pulls are in balance. Any infinitesimal body at any point of the Lagrangian points would be held there without getting pulled closer to either of massive bodies. The points $\mathrm{L}_{1}, \mathrm{~L}_{2}, \mathrm{~L}_{3}$ are colinear with the line joining the two massive bodies, while the triangular points $\mathrm{L}_{4}, \mathrm{~L}_{5}$ are found $60^{\circ}$ ahead of and behind the less massive body, along its orbit. These two triangular points $\mathrm{L}_{4,5}$ are forming equilateral triangles with the two massive bodies. The Restricted Three Body Problem (RTBP in brief) is now defined as a system consisting of two massive bodies, called the primaries, revolving in a circular orbits around their center of mass and a third body of infinitesimally small mass which moves in the primaries' orbital plane. The three collinear equilibria at $\mathrm{L}_{1}, \mathrm{~L}_{2}, \mathrm{~L}_{3}$ are unstable, while the two triangular solutions $\mathrm{L}_{4}, \mathrm{~L}_{5}$ are stable, in the linear analysis, for only a certain values of the mass ratio in the interval $(0, \mu)$ where $\mu=0.038521$ is the Routhian which modifies when including different perturbations (cf. Szebehely, 1967; Subba and Sharma, 1994; 1997; 1988; 1986; Narayan and Ramesh, 2011). The global stability of these points have been studied by several authors: (Leontovich, 1962; Deprit and Deprit-Bartholome, 1967; Markeev, 1969; Szebehely 1979; Narayan and Ramesh, 2008; Singh, 2011; Kumar and Ishwar, 2011; Douskos, 2011; Shankaran et al., 2011). Their final conclusions are that in the planar case the triangular points $\mathrm{L}_{4,5}$ are always stable within some domain of mass ratio which is modified when including such kind of different perturbations.

In this study the author is searching for new equilirium points in the peripindicular planes to the plane of motion of the primaries. We follow the same very well known way in RTBP but with the required modifications. We first compute their positions of such equilibrium points and then study their stability.

Equations of motion: The equations of motion of an infinitesimal mass in the relativistic RTBP in a synodic frame of reference $(\xi, \eta, \xi)$, in which the primaries coordinates on the $\mathrm{x}$-axis $(-\mu, 0,0),(1-\mu, 0,0)$ are kept fixed and the origin at the center of mass, are given Eq. 1 by Brumberg (1972): 


$$
\begin{aligned}
& \ddot{\xi}-2 n \eta=\frac{\partial U}{\partial \xi}-\frac{d}{d t}\left(\frac{\partial U}{\partial \dot{\xi}}\right) \\
& \ddot{\eta}+2 n \dot{\xi}=\frac{\partial U}{\partial \eta}-\frac{d}{d t}\left(\frac{\partial U}{\partial \dot{\eta}}\right) \\
& \ddot{\zeta}=\frac{\partial U}{\partial \zeta}
\end{aligned}
$$

where $n=1$ is the mean motion of the rotating system and $U$ is the potential-like (pseudopotential) function of the RTBP is given by Eq. 2 :

$$
\mathrm{U}=\frac{\mathrm{r}^{2}}{2}+\frac{1-\mu}{\mathrm{r}_{1}}+\frac{\mu}{\mathrm{r}_{2}}
$$

And:

$$
\begin{aligned}
& r_{1}^{2}=(\xi+\mu)^{2}+\eta^{2}+\zeta^{2} \\
& r_{2}^{2}=(\xi+\mu-1)^{2}+\eta^{2}+\zeta^{2} \\
& r^{2}=\xi^{2}+\eta^{2}+\zeta^{2}
\end{aligned}
$$

Equilibrium points: From the equations of motion (1), it is apparent an equilibrium solution exists relative to the rotating frame when the partial derivatives of the pseudopotential function are all zero, i.e., these points correspond to the positions in the rotating frame at which the gravitational forces and the centrifugal force associated with the rotation of the synodic frame all cancel, with the result that a particle located at one of these points appears stationary in the synodic frame.

The libration points are obtained from equations of motion (1) after setting $\ddot{\xi}=\dot{\xi}=\ddot{\eta}=\ddot{\eta}=\ddot{\zeta}=\dot{\zeta}=0$. These points represent particular solutions of equations of motion Eq. 3:

$$
\frac{\partial \mathrm{U}}{\partial \xi}=\frac{\partial \mathrm{U}}{\partial \eta}=\frac{\partial \mathrm{U}}{\partial \zeta}=0
$$

Equilibrium Points in the plane $\boldsymbol{\eta}=\mathbf{0}$ : The author expects a new set of equilibrium points in the $\xi \xi$ plane. Now we can rename the triangular Lagrange equilibrium points $L_{4}, L_{5}$ to become $L_{4}^{\xi \eta}, L_{5}^{\xi \eta}$ this because Lagrange found them in $\xi \eta$. In this study the author found a new set of equilibrium points in the $\xi \xi$ plane. This new set will be called in the future works Fawzy $\xi \xi$-triangular equilibrium points $\mathrm{L}_{4}^{\xi \zeta}, \mathrm{L}_{5}^{\xi \zeta}$. To calculate the locations of these new equilibrium points, rewrite the potential-like function $\mathrm{U}$ in a different form. By definition, this new set has $\eta=0$ and Eq. 4:

$$
\left.\begin{array}{l}
\mathrm{r}_{1}^{2}=(\xi+\mu)^{2}+\zeta^{2} \\
\mathrm{r}_{2}^{2}=(\xi+\mu-1)^{2}+\zeta^{2}
\end{array}\right\}
$$

Thus we can write Eq. 5:

$$
\begin{array}{r}
(1-\mu)\left((\xi+\mu)^{2}+\zeta^{2}\right)+\mu\left((\xi+\mu-1)^{2}+\zeta^{2}\right) \\
=\xi^{2}+\zeta^{2}-\mu(\mu-1) \\
\mathrm{U}=\frac{1}{2}(1-\mu)\left(\mathrm{r}_{1}^{2}+\frac{2}{\mathrm{r}_{1}}\right)+\frac{1}{2} \mu\left(\mathrm{r}_{2}^{2}+\frac{2}{\mathrm{r}_{2}}\right) \\
+\frac{1}{2} \mu(\mu-1)
\end{array}
$$

The system (3) can be written as Eq. 6:

$$
\left[\begin{array}{l}
\frac{\partial \mathrm{U}}{\partial \xi} \\
\frac{\partial \mathrm{U}}{\partial \zeta}
\end{array}\right]=\left[\begin{array}{ll}
(1-\mu)\left(2 \mathrm{r}_{1}-\frac{2}{\mathrm{r}_{1}^{2}}\right) & \mu\left(2 \mathrm{r}_{2}-\frac{2}{\mathrm{r}_{2}^{2}}\right) \\
(1-\mu)\left(2 \mathrm{r}_{1}-\frac{2}{\mathrm{r}_{1}^{2}}\right) & \mu\left(2 \mathrm{r}_{2}-\frac{2}{\mathrm{r}_{2}^{2}}\right)
\end{array}\right]\left[\begin{array}{l}
\frac{\partial \mathrm{r}_{1}}{\partial \xi} \\
\frac{\partial \mathrm{r}_{2}}{\partial \zeta}
\end{array}\right]=\left[\begin{array}{l}
0 \\
0
\end{array}\right]
$$

This system is verified when:

$$
(1-\mu)\left(2 r_{1}-\frac{2}{r_{1}^{2}}\right)=0, \mu\left(2 r_{2}-\frac{2}{r_{2}^{2}}\right)=0
$$

from which one can easily obtain $r_{1}=r_{2}=1$. Substituting these results back to the Eq. 4 yields:

$(\xi+\mu)^{2}+\zeta^{2}=1,(\xi+\mu-1)^{2}+\zeta^{2}=1$

Solving these two equations simultaneously yields Eq. 7:

$\xi=\frac{1}{2}-\mu, \zeta= \pm \frac{\sqrt{3}}{2}$

As expected from the symmetry of the problem, we obtained the locations of the new equilibrium points. It looks similar to Lagrange triangular equilibrium points $(\xi, \eta)=\left(\frac{1}{2}-\mu, \frac{\sqrt{3}}{2}\right),\left(\frac{1}{2}-\mu,-\frac{\sqrt{3}}{2}\right)$ but except the plane in which they lie: i.e., in the plane $\eta=0$ Eq. 8:

$$
(\xi, \zeta)=\left(\frac{1}{2}-\mu, \frac{\sqrt{3}}{2}\right),\left(\frac{1}{2}-\mu,-\frac{\sqrt{3}}{2}\right)
$$


Equilibrium points in the plane parallel to $\xi=0$ plane: We consider a test particle at a plane parallel to the plane $\xi=0$ (except at planes $\xi=-\mu$ and $\xi=1-\mu$ because we did not find any more equilibrium points there). According to these constraints we have Eq. 9:

$$
\left.\begin{array}{l}
\mathrm{r}_{2}^{2}=(\xi+\mu-1)^{2}+\eta^{2}+\zeta^{2} \\
\mathrm{r}_{1}^{2}=(\xi+\mu)^{2}+\eta^{2}+\zeta^{2} \\
\mathrm{r}^{2}=\xi^{2}+\eta^{2}+\zeta^{2}
\end{array}\right\}
$$

Thus we can write:

$$
\begin{aligned}
& (1-\mu)\left((\xi+\mu)^{2}+\eta^{2}+\zeta^{2}\right) \\
& +\mu\left((\xi+\mu-1)^{2}+\eta^{2}+\zeta^{2}\right)= \\
& \xi^{2}+\eta^{2}+\zeta^{2}-\mu(\mu-1)
\end{aligned}
$$

Substituting into (2):

$$
\begin{aligned}
& \mathrm{U}=\frac{1}{2}(1-\mu)\left(\mathrm{r}_{1}^{2}+\frac{2}{\mathrm{r}_{1}}\right)+\frac{1}{2} \mu\left(\mathrm{r}_{2}^{2}+\frac{2}{\mathrm{r}_{2}}\right) \\
& +\frac{1}{2} \mu(\mu-1)
\end{aligned}
$$

The system (3) can be written as Eq. 10:

$$
\left.\begin{array}{l}
\frac{\partial \mathrm{U}}{\partial \xi}=\sum_{\mathrm{i}=1}^{2} \frac{\partial \mathrm{U}}{\partial \mathrm{r}_{\mathrm{i}}} \frac{\partial \mathrm{r}_{\mathrm{i}}}{\partial \xi}=(1-\mu)\left(2 \mathrm{r}_{1}-\frac{2}{\mathrm{r}_{1}^{2}}\right) \frac{(\xi+\mu)}{\mathrm{r}_{1}} \\
+\mu\left(2 \mathrm{r}_{2}-\frac{2}{\mathrm{r}_{2}^{2}}\right) \frac{(\xi+\mu-1)}{\mathrm{r}_{2}}=0 \\
\frac{\partial \mathrm{U}}{\partial \eta}=\sum_{\mathrm{i}=1}^{2} \frac{\partial \mathrm{U}}{\partial \mathrm{r}_{1}} \frac{\partial \mathrm{r}_{1}}{\partial \eta}=(1-\mu)\left(2 \mathrm{r}_{1}-\frac{2}{\mathrm{r}_{1}^{2}}\right) \frac{\eta}{\mathrm{r}_{1}} \\
+\mu\left(2 \mathrm{r}_{2}-\frac{2}{\mathrm{r}_{2}^{2}}\right) \frac{\eta}{\mathrm{r}_{2}}=0 \\
\frac{\partial \mathrm{U}}{\partial \zeta}=\sum_{\mathrm{i}=1}^{2} \frac{\partial \mathrm{U}}{\partial \mathrm{r}_{\mathrm{i}}} \frac{\partial \zeta}{\partial \mathrm{r}_{i}}=(1-\mu)\left(2 \mathrm{r}_{1}-\frac{2}{\mathrm{r}_{1}^{2}}\right) \frac{\zeta}{\mathrm{r}_{1}} \\
+\mu\left(2 \mathrm{r}_{2}-\frac{2}{\mathrm{r}_{2}^{2}}\right) \frac{\zeta}{\mathrm{r}_{2}}=0
\end{array}\right\}
$$

This system is verified when $\frac{\partial \mathrm{U}}{\partial \mathrm{r}_{1}}=0, \frac{\partial \mathrm{U}}{\partial \mathrm{r}_{2}}=0 \mathrm{Eq} .11$

$$
(1-\mu)\left(2 \mathrm{r}_{1}-\frac{2}{\mathrm{r}_{1}^{2}}\right)=0, \mu\left(2 \mathrm{r}_{2}-\frac{2}{\mathrm{r}_{2}^{2}}\right)=0
$$

from which one can easily obtain $r_{1}=r_{2}=1$. Substituting these results back to the Eq. 9 yields Eq. 12:

$$
\left.\begin{array}{l}
(\xi+\mu)^{2}+\eta^{2}+\zeta^{2}=1 \\
(\xi+\mu-1)^{2}+\eta^{2}+\zeta^{2}=1
\end{array}\right\}
$$

Solving these two equations simultaneously for $\xi$ yields $\xi=\frac{1}{2}-\mu$, substituting back into Eq. 8 we get $\eta^{2}+\zeta^{2}=\frac{3}{4} \Rightarrow \zeta= \pm \sqrt{\frac{3}{4}-\eta^{2}}$ provided that $\|\eta\| \leq \frac{\sqrt{3}}{2} \Rightarrow$ $\frac{-\sqrt{3}}{2} \leq \eta \leq \frac{\sqrt{3}}{2}$. Therefore we have infinite number of solutions (equilibrium points), their coordinates are given by Eq. 13:

$$
(\xi, \eta, \zeta)=\left(\frac{1}{2}-\mu, \frac{-\sqrt{3}}{2} \leq \eta \leq \frac{\sqrt{3}}{2} \leq, \zeta= \pm \sqrt{\frac{3}{4}-\eta^{2}}\right)
$$

Remark 1: As is clear from the solution set we have obtained infinte number of equilibrium points which lie on a circumference of a circle centered at $\left(\frac{1}{2}-\mu, 0,0\right)$ and its circumference passes through the Lagrange and Fawzy triangular equilibrium points $\mathrm{L}_{4}^{\xi \eta}, \mathrm{L}_{5}^{\xi \eta}, \mathrm{L}_{4}^{\xi \zeta}, \mathrm{L}_{5}^{\xi \zeta}$. This circle will be called in the future works Fawzy equilibrium circle. i.e., The Lagrange and Fawzy triangular equilibrium points is a subset of Fawzy equilibrium circle.

Special cases: To check our solutions, setting $\eta= \pm \frac{\sqrt{3}}{2}$ in (13) yields directly the familiar Lagrangian triangular equilibrium points $L_{4}^{\xi \eta}, L_{5}^{\xi \eta}$ on respective at $(\xi, \eta)=\left(\frac{1}{2}-\mu, \pm \frac{\sqrt{3}}{2}\right)$. Also if we set $\eta=0$ Fawzy triangular equilibrium points follow directly as $\mathrm{L}_{4}^{\xi \zeta}, \mathrm{L}_{5}^{\xi \zeta}$ on respective at $(\xi, \zeta)=\left(\frac{1}{2}-\mu, \pm \frac{\sqrt{3}}{2}\right)$.

Stability of fawzy equilibrium circle: We are going to study the stability of Fawzy equilibrium circle. At each step we will examine our solution using the well known results in RTBP. To examine the stability, an infinitesimal body would be displaced a little from the Fawzy equilibrium circle. If the resultant motion of the particle is a rapid departure from the vicinity of this circle we can call such a circle an unstable circle, if however the particle merely oscillates about the equilibrium points of its circumference, it is said to be a stable positions. If we have a mixed situation then we 
will determine the stable points and the unstable ones. To examine the stability of the orbits in the vicinity of Fawzy equilibrium circle the equations of motion are linearized around the circumference of the equilibrium circle with coordinates Eq. 14:

$$
\begin{aligned}
& \xi_{0}=\eta_{0}=\zeta_{0}=\dot{\xi}_{0}=\dot{\eta}_{0}=\dot{\zeta}_{0}=0 \\
& \ddot{\xi}_{0}-2 \mathrm{n} \dot{\eta}_{0}=\left(\frac{\partial \mathrm{U}}{\partial \xi}\right)_{\xi=\xi_{0}} \\
& \ddot{\eta}_{0}+2 n \dot{\xi}_{0}=\left(\frac{\partial U}{\partial \eta}\right)_{\eta=\eta_{0}} \\
& \ddot{\zeta}_{0}=\left(\frac{\partial \mathrm{U}}{\partial \zeta}\right)_{\zeta=\zeta_{0}}
\end{aligned}
$$

the subscript 0 indicates evaluation for $\xi=\xi_{0}, \eta=\eta_{0}$ and $\xi=\xi_{0}$. If Eq. 14 are now evaluated at $\xi=\xi_{0}+\xi_{1}$, $\xi$ $=\xi_{0}+\xi_{1}$ and $\eta=\eta_{0}+\eta_{1}$, one can get Eq. 15:

$$
\left.\begin{array}{l}
\ddot{\xi}_{1}-2 \mathrm{n} \eta_{1}=\left(\mathrm{U}_{\xi \xi}\right) \xi_{1}+\left(\mathrm{U}_{\xi \eta}\right) \eta_{1}+\left(\mathrm{U}_{\xi \zeta}\right) \zeta_{1}+\ldots \\
\ddot{\eta}_{1}+2 \mathrm{n} \dot{\xi}_{1}=\left(\mathrm{U}_{\xi \eta}\right) \xi_{1}+\left(\mathrm{U}_{\eta \eta}\right) \eta_{1}+\left(\mathrm{U}_{\eta \zeta}\right) \zeta_{1}+\ldots \\
\ddot{\zeta}_{1}=\left(\mathrm{U}_{\zeta \xi}\right) \xi_{1}+\left(\mathrm{U}_{\zeta \eta}\right) \eta_{1}+\left(\mathrm{U}_{\zeta \zeta}\right) \zeta_{1}+\ldots
\end{array}\right\}
$$

We can rewrite Eq. 15 in matrix notation as Eq. 16:

$$
\left[\begin{array}{l}
\dot{\xi}_{1} \\
\dot{\eta}_{1} \\
0 \\
\ddot{\xi}_{1} \\
\ddot{\eta}_{1} \\
\ddot{\zeta}_{1}
\end{array}\right]=\left[\begin{array}{llllll}
0 & 0 & 0 & 1 & 0 & 0 \\
0 & 0 & 0 & 0 & 1 & 0 \\
0 & 0 & 0 & 0 & 0 & 0 \\
U_{\xi \xi} & U_{\xi \eta} & U_{\xi \zeta} & 0 & 2 & 0 \\
U_{\xi \eta} & U_{\eta \eta} & U_{\eta \zeta} & -2 & 0 & 0 \\
U_{\zeta \xi} & U_{\zeta \eta} & U_{\zeta \zeta} & 0 & 0 & 0
\end{array}\right]\left[\begin{array}{l}
\xi_{1} \\
\eta_{1} \\
\zeta_{1} \\
\dot{\xi}_{1} \\
\dot{\eta}_{1} \\
0
\end{array}\right]
$$

The point is switching from the solution of simultaneous second order differential equations to the solution of a system of first order differential equations. Let us denote to the state vector by $\left(X \equiv \xi_{1}, \eta_{1}, \zeta_{1}, \dot{\xi}_{1}, \dot{\eta}_{1}\right)$ accordingly $\dot{\mathrm{X}}$ represents any of $\dot{\xi}_{1}, \dot{\eta}_{1}, \ddot{\xi}_{1}, \ddot{\eta}_{1}, \ddot{\zeta}_{1}$, thus Eq. 16 becomes Eq. 17:

$$
\dot{\mathrm{X}}=\mathrm{AX}
$$

To solve this system we compute first the eigenvalues of the coefficient matrix A as follows Eq. 18:

$$
(\mathrm{A}-\lambda \mathrm{I}) \mathrm{X}=0 \Rightarrow \operatorname{det}(\mathrm{A}-\lambda \mathrm{I})=0
$$

Therefore we get the characteristic equation, with degree $\mathrm{n}$ in $\lambda$ with possible complex roots. After getting the eigenvalues $\lambda$ 's substitute them into (A$\lambda \mathrm{I}) \mathrm{X}=0$ and solve for the components $\mathrm{X}$. The resulting equations are coupled (different components are appeared in the same equation). Using the similarity transformation $\mathrm{Y}=\mathrm{BX}$ we can transform this coupled system to uncoupled one as:

$$
\begin{aligned}
& \mathrm{Y}=\mathrm{BX} \Rightarrow \mathrm{X}=\mathrm{B}^{-1} \mathrm{Y} \Rightarrow \dot{\mathrm{X}}=\mathrm{B}^{-1} \dot{\mathrm{Y}} \\
& \Rightarrow \mathrm{B}^{-1} \dot{\mathrm{Y}}=\mathrm{AB}^{-1} \mathrm{Y} \Rightarrow \dot{\mathrm{Y}}=\mathrm{BAB}^{-1} \mathrm{Y}
\end{aligned}
$$

where, $\mathrm{BAB}^{-1}$ is a diagonal matrix with the eigenvalues of $\mathrm{A}$ on the diagonal, the matrix $\mathrm{B}$ is constructed from the $\mathrm{n}$ eigenvalues the matrix A. Now The coupled system (17) is transformed to uncoupled Eq. 19:

$\dot{\mathrm{Y}}_{\mathrm{i}}=\mathrm{BAB}^{-1} \mathrm{Y}_{\mathrm{i}}=\lambda_{\mathrm{i}} \mathrm{Y}_{\mathrm{i}}$

The solutions of the transformed system are easily found as Eq. 20:

$$
\mathrm{Y}_{\mathrm{i}}=\mathrm{c}_{\mathrm{i}} \exp \left(\lambda_{\mathrm{i}} \mathrm{t}\right)
$$

where $c_{i}$ are $n$ constants of integration. We must now transform back to the original variables in components form as Eq. 21 and 22:

$$
\begin{aligned}
& \mathrm{X}_{\mathrm{i}}=\mathrm{B}^{-1} \mathrm{Y}_{\mathrm{i}}=\mathrm{B}^{-1} \mathrm{c}_{\mathrm{i}} \exp \left(\lambda_{\mathrm{i}} \mathrm{t}\right) \\
& {\left[\begin{array}{l}
\dot{\xi}_{1} \\
\dot{\eta}_{1} \\
0 \\
\ddot{\xi}_{1} \\
\ddot{\eta}_{1} \\
\ddot{\zeta}_{1}
\end{array}\right]=\left[\begin{array}{llllll}
\lambda & 0 & 0 & 1 & 0 & 0 \\
0 & \lambda & 0 & 0 & 1 & 0 \\
0 & 0 & \lambda & 0 & 0 & 0 \\
\mathrm{U}_{\xi \xi} & \mathrm{U}_{\xi \eta} & \mathrm{U}_{\xi \zeta} & \lambda & 2 & 0 \\
\mathrm{U}_{\xi \eta} & \mathrm{U}_{\eta \eta} & \mathrm{U}_{\eta \zeta} & -2 & \lambda & 0 \\
\mathrm{U}_{\zeta \xi} & \mathrm{U}_{\zeta \eta} & \mathrm{U}_{\zeta \zeta} & 0 & 0 & \lambda
\end{array}\right]\left[\begin{array}{l}
\xi_{1} \\
\eta_{1} \\
\zeta_{1} \\
\dot{\xi}_{1} \\
\dot{\eta}_{1} \\
0
\end{array}\right]} \\
& \operatorname{det}(\mathrm{A}-\lambda \mathrm{I})=\left[\begin{array}{llllll}
-\lambda & 0 & 0 & 1 & 0 & 0 \\
0 & -\lambda & 0 & 0 & 1 & 0 \\
0 & 0 & -\lambda & 0 & 0 & 0 \\
\mathrm{U}_{\xi \xi} & \mathrm{U}_{\xi \eta} & \mathrm{U}_{\xi \zeta} & -\lambda & 2 & 0 \\
\mathrm{U}_{\xi \eta} & \mathrm{U}_{\eta \eta} & \mathrm{U}_{\eta \zeta} & -2 & -\lambda & 0 \\
\mathrm{U}_{\zeta \xi} & \mathrm{U}_{\zeta \eta} & \mathrm{U}_{\zeta \zeta} & 0 & 0 & -\lambda
\end{array}\right]=0
\end{aligned}
$$

which are linear differential equations with constant coefficients so long as only first order terms are retained. Let a solution of the problem be Eq. 23: 
Am. J. Applied Sci., 9 (9): 1378-1384, 2012

$$
\left.\begin{array}{l}
\xi_{1}=A \exp (\lambda t) \\
\eta_{1}=B \exp (\lambda t) \\
\zeta_{1}=C \exp (\lambda t)
\end{array}\right\}
$$

where, $\mathrm{A}, \mathrm{B}, \mathrm{C}$ and $\lambda$ are constants. To find the expressions for $\mathrm{A}, \mathrm{B}$ and $\mathrm{C}$ Eq. 15 can be rewritten, using the suggested solution, as Eq. 24:

$$
\begin{aligned}
& \left(\lambda^{2}-\mathrm{U}_{\xi \xi}\right) \mathrm{A}-\left(2 \lambda+\mathrm{U}_{\xi \eta}\right) \mathrm{B}-\left(\mathrm{U}_{\xi \xi}\right) \mathrm{C}=0 \\
& \left.\left(2 \lambda-U_{\xi \eta}\right) A+\left(\lambda^{2}-U_{\eta \eta}\right) B-\left(U_{\eta \zeta}\right) C=0\right\} \\
& -\left(\mathrm{U}_{\zeta \xi}\right) \mathrm{A}-\left(\mathrm{U}_{\zeta \eta}\right) \mathrm{B}+\left(\lambda^{2}-\mathrm{U}_{\zeta \zeta}\right) \mathrm{C}=0
\end{aligned}
$$

which can be written in matrix notation as Eq. 25:

$$
\left[\begin{array}{lll}
\lambda^{2}-\mathrm{U}_{\xi \xi} & -\left(2 \lambda+\mathrm{U}_{\xi \eta}\right) & -\left(\mathrm{U}_{\xi \zeta}\right) \\
\left(2 \lambda-\mathrm{U}_{\xi \eta}\right) & \left(\lambda^{2}-\mathrm{U}_{\eta \eta}\right) & -\left(\mathrm{U}_{\eta \zeta}\right) \\
-\left(\mathrm{U}_{\zeta \xi}\right) & -\left(\mathrm{U}_{\zeta \eta}\right) & \left(\lambda^{2}-\mathrm{U}_{\zeta \zeta}\right)
\end{array}\right]\left[\begin{array}{l}
\mathrm{A} \\
\mathrm{B} \\
\mathrm{C}
\end{array}\right]=\left[\begin{array}{l}
0 \\
0 \\
0
\end{array}\right]
$$

This system has nontrivial solution if Eq. 26:

$$
\left|\begin{array}{lll}
\lambda^{2}-U_{\xi \xi} & -\left(2 \lambda+U_{\xi \eta}\right) & -\left(U_{\xi \zeta}\right) \\
\left(2 \lambda-U_{\xi \eta}\right) & \left(\lambda^{2}-U_{\eta \eta}\right) & -\left(U_{\eta \zeta}\right) \\
-\left(U_{\zeta \xi}\right) & -\left(U_{\zeta \eta}\right) & \left(\lambda^{2}-U_{\zeta \zeta}\right)
\end{array}\right|=0
$$

expanding the determinant yields Eq. 27:

$$
\begin{aligned}
& 0=\lambda^{6}+\left(4-U_{\xi \xi}-U_{\eta \eta}-U_{\zeta \zeta}\right) \lambda^{4} \\
& +\left(U_{\xi \xi} U_{\eta \eta}+U_{\xi \xi} U_{\zeta \zeta}+U_{\eta \eta} U_{\zeta \zeta}\right. \\
& \left.-4 U_{\zeta \zeta}-U_{\xi \eta}^{2}-U_{\xi \zeta}^{2}-U_{\eta \zeta}^{2}\right) \lambda^{2} \\
& +\left(-U_{\xi \xi} U_{\eta \eta} U_{\zeta \zeta}-2 U_{\xi \eta} U_{\xi \zeta} U_{\eta \zeta}\right. \\
& \left.+U_{\xi \xi} U_{\eta \zeta}^{2}+U_{\xi \eta}^{2} U_{\zeta \zeta}+U_{\xi \zeta}^{2} U_{\eta \eta}\right)
\end{aligned}
$$

where $\sigma$ is the root of the characteristic determinant and $\mathrm{U}_{\alpha \beta}$ are the second partial derivatives with respect to the variables mentioned in the subscripts and evaluated at the equilibrium points. Evaluating the partial derivatives included in Eq. 27. Now computing the coefficients of $\lambda$ 's, then the characteristic Eq. 27 can be written in the form Eq. 28:

$$
\begin{aligned}
& \lambda^{6}+\lambda^{4}+\left(9 \mu(1-\mu)\left(\eta^{2}+\zeta^{2}\right)-12 \zeta^{2}\right) \lambda^{2} \\
& +108 \xi^{2} \eta^{2} \zeta^{2}=0
\end{aligned}
$$

To check at this step let us return back to the stability investigation in the case of Lagrange's triangular points: (setting $\zeta=0, \eta=\frac{\sqrt{3}}{2}$ ) we ge Eq. 29t:

$$
\begin{aligned}
& \lambda^{6}+\lambda^{4}+\frac{27}{4} \mu(1-\mu) \lambda^{2}=0 \\
& \Rightarrow \lambda^{4}+\lambda^{2}+\frac{27}{4} \mu(1-\mu)=0
\end{aligned}
$$

which has the four solutions:

$$
\lambda_{1,2,3,4}= \pm \frac{1}{\sqrt{2}} \sqrt{-1 \pm \sqrt{27 \mu^{2}-27 \mu+1}}
$$

The only oscillatory stable solution when $\lambda^{2}$ is real and negative. In this case two purely imaginary roots $\lambda= \pm i \sqrt{\left|\lambda^{2}\right|}$ exist. This case is verified when:

$$
27 \mu^{2}-27 \mu+1 \leq 0
$$

i.e., when the mass ratio becomes the very well known result $\mu=0.038521$. Now return back to characteristic Eq. 27. It has the solutions Eq. 30:

$$
\begin{aligned}
& \lambda= \pm \frac{1}{\sqrt{2}}\left[\left[-\left(9 \mu(1-\mu)\left(\eta^{2}+\zeta^{2}\right)-12 \zeta^{2}\right)\right.\right. \\
& \pm\left(9 \mu(1-\mu)\left(\eta^{2}+\zeta^{2}\right)-12 \zeta^{2}\right)^{2} \\
& \left.\left.-108(1-2 \mu)^{2} \eta^{2} \zeta^{2}\right]^{\frac{1}{2}}\right]^{\frac{1}{2}}
\end{aligned}
$$

\section{$\eta$-Dependent Stability:}

Setting:

$$
\zeta^{2}=\frac{3}{4}-\eta^{2}, \xi^{2}=\left(\frac{1}{2}-\mu\right)^{2}
$$

Yields Eq. 31:

$$
\begin{aligned}
& \lambda= \pm \frac{1}{\sqrt{2}}\left[-\left(\frac{27}{4} \mu(1-\mu)+12 \eta^{2}-9\right)\right. \\
& \pm\left[\left(\frac{27}{4} \mu(1-\mu)+12 \eta^{2}-9\right)^{2}\right. \\
& \left.\left.-27(1-2 \mu)^{2}\left(\eta^{2}-4 \eta^{4}\right)\right]^{\frac{1}{2}}\right]^{\frac{1}{2}}
\end{aligned}
$$

The quantity $\frac{27}{4} \mu(1-\mu)$ is positive definite and the quantity $12 \eta^{2}-9 \geq 0$, only when: 


$$
\eta \in\left(-\infty,-\frac{\sqrt{3}}{2}\right] \cup\left[\frac{\sqrt{3}}{2}, \infty\right)
$$

The quantity inside the inner square root can be checked for the negative values as follows; setting:

$$
F(\eta)=\left(\frac{27}{4} \mu(1-\mu)+12 \eta^{2}-9\right)^{2}-27(1-2 \mu)^{2}\left(\eta^{2}-4 \eta^{4}\right)
$$

Expanding F ( $\eta$ ) yields Eq. 32:

$$
\begin{aligned}
& \mathrm{F}(\eta)=\left(\frac{2673}{16} \mu^{2}-\frac{243}{2} \mu-\frac{729}{8} \mu^{3}+\frac{729}{16} \mu^{4}+81\right) \\
& +\left(-243+270 \mu-270 \mu^{2}\right) \eta^{2} \\
& +\left(252-432 \mu+432 \mu^{2}\right) \eta^{4}
\end{aligned}
$$

which can be written as $F(\eta)=a_{0}+a_{1} \eta^{2}+a_{2} \eta^{4}$, where $a_{0} \geq 0, a_{1} \leq 0, a_{2} \geq 0$ are the coefficients of $\eta$ 's in Eq. 32 . Completing the square and searching for the negative values yields:

$$
\begin{aligned}
F(\eta) & =a_{0}+\left(\sqrt{a_{2}} \eta^{2}+\frac{a_{1}}{2 \sqrt{a_{2}}}\right)^{2}-\frac{a_{1}^{2}}{4 a_{2}}<0 \\
\Rightarrow \eta & \leq \pm\left[-\frac{a_{1}}{2 a_{2}} \pm \frac{1}{\sqrt{a_{2}}}\left[\frac{a_{1}^{2}}{4 a_{2}}-a_{0}\right]^{\frac{1}{2}}\right]^{\frac{1}{2}} \\
\eta & \leq \pm\left[-\frac{\left(-243+270 \mu-270 \mu^{2}\right)}{2\left(252-432 \mu+432 \mu^{2}\right)}\right. \\
\pm & \sqrt{\left(252-432 \mu+432 \mu^{2}\right)} \\
& \times \frac{\left(-243+270 \mu-270 \mu^{2}\right)^{2}}{4\left(252-432 \mu+432 \mu^{2}\right)} \\
& -\left(-\frac{243}{2} \mu+\frac{2673}{16} \mu^{2}-\frac{729}{8} \mu^{3}\right. \\
& \left.\left.\left.+\frac{729}{16} \mu^{4}+81\right)\right]^{\frac{1}{2}}\right]^{\frac{1}{2}}
\end{aligned}
$$

Expanding and retaining the terms up to $\mathcal{O}\left(\mu^{5}\right)$ the above equation can be rewritten in the form Eq. 33:

$$
\begin{aligned}
& \eta \leq \pm\left[\frac{27}{56}+\frac{57}{196} \mu+\frac{285}{1372} \mu^{2}\right. \\
& -\frac{342}{2401} \mu^{3}-\frac{10089}{16807} \mu^{4}-\frac{92340}{117649} \mu^{5} \\
& \pm\left[-\frac{22599}{4}+32805 \mu-\frac{314199}{4} \mu^{2}\right. \\
& \left.\left.+\frac{222345}{2} \mu^{3}-\frac{419175}{4} \mu^{4}+59049 \mu^{5}\right]^{\frac{1}{2}}\right]^{\frac{1}{2}}
\end{aligned}
$$

-Dependent stability: Recall Eq. 30 and setting $\eta^{2}=\frac{3}{4}-\zeta^{2}, \xi^{2}=\left(\frac{1}{2}-\mu\right)^{2}$ yields:

$$
\begin{aligned}
& \lambda= \pm \frac{1}{\sqrt{2}}\left[-\left(\frac{27}{4} \mu(1-\mu)-12 \zeta^{2}\right)\right. \\
& \pm\left[\left(\frac{27}{4} \mu(1-\mu)-12 \zeta^{2}\right)^{2}\right. \\
& \left.\left.-27(1-2 \mu)^{2}\left(\zeta^{2}-4 \zeta^{4}\right)\right]^{\frac{1}{2}}\right]^{\frac{1}{2}}
\end{aligned}
$$

The quantity $\left(\frac{27}{4} \mu(1-\mu)-12 \zeta^{2}\right)$ is positive when:

$$
\zeta \in\left(\frac{3}{4} \sqrt{\mu(1-\mu)}, \infty\right) \cup\left(-\infty,-\frac{3}{4} \sqrt{\mu(1-\mu)}\right)
$$

The quantity inside the inner square root can be checked for the negative values as follows; setting:

$$
\begin{aligned}
& \mathrm{F}(\zeta)=\left(\frac{27}{4} \mu(1-\mu)-12 \zeta^{2}\right)^{2} \\
& -27(1-2 \mu)^{2}\left(\zeta^{2}-4 \zeta^{4}\right)
\end{aligned}
$$

Expanding $\mathrm{F}(\zeta)$ yields Eq. 34:

$F(\xi)=\frac{729}{16} \mu^{2}\left(1-2 \mu+\mu^{4}\right)+162 \mu(\mu-1) \xi^{2}+14 \xi^{4}$

which can be written as $F(\zeta)=a_{0}+a_{1} \zeta^{2}+a_{2} \zeta^{4}$, where $\mathrm{a}_{0} \geq 0, \mathrm{a}_{1}<0, \mathrm{a}_{2} \geq 0$ are the coefficients of $\zeta$ 's in Eq. 34 . Completing the square and searching for the negative values yields: 


$$
\begin{aligned}
& F(\zeta)=a_{0}+\left(\sqrt{a_{2}} \zeta^{2}+\frac{a_{1}}{2 \sqrt{a_{2}}}\right)^{2}-\frac{a_{1}^{2}}{4 a_{2}}<0 \\
& \Rightarrow \zeta \leq \pm\left[-\frac{a_{1}}{2 a_{2}} \pm \frac{1}{\sqrt{a_{2}}}\left[\frac{a_{1}^{2}}{4 a_{2}}-a_{0}\right]^{\frac{1}{2}}\right]^{\frac{1}{2}}
\end{aligned}
$$

Thus Eq. 35:

$$
\begin{aligned}
& \zeta \leq \pm \frac{1}{12}\left[\left(81 \pm \frac{3 \sqrt{3}}{2}\right) \mu(1-\mu)\right. \\
& \left.-\frac{3 \sqrt{3}}{256} \mu^{3}\left(16+8 \mu+\mu^{3}\right)\right]^{\frac{1}{2}}
\end{aligned}
$$

As is clear from Eq. 33 and 35 the general solution for the components of the position and velocity around the equilibrium points of on the circumference of the circle involve a linear combinations, linearly independent terms, of $\exp \left(+\mathrm{i} \sqrt{\left|\lambda^{2}\right|} \mathrm{t}\right)$ and $\exp \left(-\mathrm{i} \sqrt{\left|\lambda^{2}\right|} \mathrm{t}\right)$ that leads by Euler identity to sines and cosines. Thus we obtained oscillatory stable solutions when $\eta$ or $\xi$ satisfies the critria derived in (33) and (35).

\section{ACKNOWLEDGMENTS}

The author is deeply indebted and thankful for the deanship of the scientific research for his helpful and for distinct team of the employees at Taibah university, Al-Madinah Al-Munawwarah, K.S.A. This research study was supported by a grant No. (164/1332).

\section{REFERENCES}

Brumberg, V.G., 1972. Relativistic celestial mechanics. Nauka, Moscow.

Deprit, A. and A. Deprit-Bartholome, 1967. Stability of the triangular lagrangian points. Astron. J., 72: 173-179.

Douskos, C.N., 2011. Equilibrium points of the restricted three-body problem with equal prolate and radiating primaries and their stability. Astrophys Space Sci., 333: 79-87. DOI: 10.1007/s10509-010-0584-7

Kumar, S. and B. Ishwar, 2011. Location of collinear equilibrium points in the generalised photogravitational elliptic restricted three body problem. Int. J. Eng., Sci. Technol., 3: 157-162.
Leontovich, A.M., 1962. On the stability of the restricted problem of three bodies. Soviet math. Dokl., 3: 425-428.

Markeev, A.P., 1969. On the stability of the triangular libration points in the circular bounded three-body problem. J. Applied Math. Mech., 33: 105-110. DOI: $10.1016 / 0021-8928(69) 90117-8$

Narayan, A. and C. Ramesh, 2008. Stability of triangular points in the generalized restricted three body problem. J. Mod. Ex-B, France.

Narayan, A. and C.R. Ramesh, 2011. Stability of triangular equilibrium points in elliptical restricted three body problem under the effects of photogravitational and oblateness of primaries. Int. J. Pure Applied Math., 70: 735-754.

Shankaran, S., J.P. Sharma and B. Ishwar, 2011. Equilibrium points in the generalised photogravitational non-planar restricted three body problem. Int. J. Eng., Sci. Technol., 3: 63-67.

Sharma, R.K. and R.P.V. Subba, 1986. On finite periodic orbits around the equilateral solutions of the planar restricted three-body problem. Proceedings of the International Workshop Space Dynamics and Celestial Mechanics, Nov. 14-16, Dordrecht, D. Reidel Publishing Co., Delhi, India, pp: 71-85.

Singh, J., 2011. Nonlinear stability in the restricted three-body problem with oblate and variable mass. Astrophys Space Sci., 333: 61-69. DOI: 10.1007/s10509-010-0572-y

Subba, R.P.V. and R.K. Sharma, 1988. Oblateness effect on finite periodic orbits at $\mathrm{L}_{4}$. Proceedings of the 39th IAF, International Astronautical Congress, Oct. 8-15, Bangalore, India, pp: 6.

Subba, R.P.V. and R.K. Sharma, 1994, Stability of $\mathrm{L}_{4}$ in the Restricted Three-Body Problem with Oblateness. 1st Edn., Vikram Sarabhai Space Centre, India, pp: 34.

Subba, R.P.V. and R.K. Sharma, 1997. Effect of oblateness on the non-linear stability of in the restricted three-body problem. Celest. Mech. Dyn. Astr. 65: 291-312.

Szebehely, V.G., 1967. Theory of Orbits in the Restricted Problem of Three Bodies. Academic Press, New York, ISBN-10: 0126806500, pp: 668.

Szebehely, V.G., 1979. Instabilities in Dynamical Systems: Applications to Celestial Mechanics. 1st Edn., D. Reidel Pub. Co., Dordrecht, ISBN: 9027709734, pp: 314. 